\title{
Experimental Research
}

\section{Parameters influencing augmentation of cerebral blood flow by cervical spinal cord stimulation}

\author{
J. Zhong, D.-L. Huang, and O. Sagher \\ Department of Neurosurgery, University of Michigan Health System, Ann Arbor, Michigan, USA
}

Received February 16, 2004; accepted July 14, 2004; published online September 3, 2004

(C) Springer-Verlag 2004

\begin{abstract}
Summary
Background. Cervical spinal cord stimulation (SCS) has been shown to augment cerebral blood flow (CBF) and protect the brain from focal ischemia. However, the application of SCS in the treatment of cerebral ischemia requires a better understanding of the limits of the cerebrovascular effect and the optimal stimulation parameters. In the present study, we investigated the effects of various stimulation parameters on $\mathrm{CBF}$ augmentation, as well as the issue of tachyphylaxis of the CBF response.

Methods. SCS was performed in adult Sprague Dawley rats, and CBF was assessed using cortical laser Doppler flowmetry (LDF). In separate experimental series, stimulation amplitude, frequency, and pulse width were varied, and the effect on the LDF response was recorded. Finally, using the stimulation parameters found to elicit the strongest LDF response, we examined the effect of lengthening the period of SCS.

Findings. SCS elicited a robust increase in cortical LDF values as previously demonstrated. The magnitude of the response varied in a dose-dependent fashion with the stimulation amplitude. LDF values increased by more than $80 \%$ over baseline with an amplitude of $1.5 \mathrm{~mA}$. The optimal pulse width and frequency of the stimulation were $0.25 \mathrm{~ms}$ and $50 \mathrm{~Hz}$, respectively. Lengthening the stimulation period up to 20 minutes resulted in a persistent increase in cerebral LDF values during the entire stimulation period, although the magnitude of this effect diminished to approximately $50 \%$ over the baseline after 10 minutes.

Conclusions. SCS elicits a robust augmentation in CBF, which lasts the entire stimulation duration. Stimulation parameters required for optimal cerebrovascular response are within normally used therapeutic ranges in the clinical settings. These results provide further evidence that SCS may provide a novel therapeutic strategy for the treatment of cerebral ischemia.
\end{abstract}

Keywords: Spinal cord stimulation; cerebral blood flow; parameter; tachyphylaxis; rat.

\section{Introduction}

Electrical stimulation of the spinal cord is an effective therapeutic modality for the treatment of various pain syndromes $[5,17,24]$. The effect of spinal cord stimulation (SCS) on peripheral blood flow has been well-described in experimental studies [19, 20] and clinical reports $[7,11,18]$, leading to the use of SCS in the treatment of painful peripheral ischemia [14, 32, 34]. The effect of SCS on peripheral vascular tone has prompted clinicians and investigators to examine the effects of stimulation on myocardial blood flow $[1,12]$ and cerebral blood flow (CBF) $[6,10,15]$. In both cases, there is mounting evidence that SCS may meaningfully increase tissue perfusion. The treatment of angina with SCS, for example, has been studied in a large clinical trial which has demonstrated significant symptomatic relief and protection from fatal myocardial events comparable to direct coronary revascularization $[2,8,13]$. Clinical experience in the use of SCS in peripheral vascular disease and angina has resulted in the widespread use of this modality for the treatment of ischemia.

The possibility that SCS may be used to augment CBF stems from the observation of Hosobuchi et al. [15], who noted that global CBF increased in a small cohort of patients treated with SCS for chronic pain. Subsequently, several investigators have demonstrated CBF changes in response to SCS in animal models. Garcia et al. [10] observed that SCS increased hemispheric blood flow with a mean of 55\% in goats and dogs as measured by laser Doppler flowmetry. Quantitative measurements with iodoantipyrine also demonstrated a significant increase in CBF. Experimentally induced cerebral ischemia has also found to be ameliorated by SCS in cats [21], rabbits [39] and rats [27, 30, 31]. 
Finally, anecdotal clinical reports of SCS have corroborated these experimental findings [22, 23].

The promise of SCS-induced CBF augmentation has led some clinicians to use this procedure in the treatment of cerebral ischemia [4]. However, the parameters used in SCS to augment CBF have been arbitrarily determined, and it is unknown whether a dose-dependent effect exists in this phenomenon. In the present study, we investigated the effects of modulating stimulation parameters on the cerebrovascular response. In addition, we examined the effects of prolonged stimulation on CBF. Such basic study is crucial and will allow more effective clinical utilization of this technique in the treatment of cerebral ischemia.

\section{Methods and materials}

\section{Animal preparation}

All experimental protocols were approved by the University of Michigan Committee on the Use and Care of Animals. Adult male Sprague-Dawley rats, each weighing between 250 and 350 grams, were selected for the experimental series. The animals were housed in standard conditions in a laboratory environment with free access to food and water. Care was taken to minimize stress and nociceptive input to the animals before and during surgery. General anesthesia was induced with 5\% isoflurane (Aerrane). After intubation and initiation of mechanical ventilation with a rodent ventilator (model 683; Harvard Apparatus, Inc., S. Natick, MA), isoflurane was titrated between $1.5-2.25 \%$ to maintain a mean arterial pressure between 80 and $120 \mathrm{mmHg}$ and a normal $\mathrm{PaCO}_{2}$ level between $35-45 \mathrm{mmHg}$. The concentration of isoflurane was held constant during the experiment. The rats were paralyzed with gallamine (Sigma Chemical Co., St. Louis, MO) $10 \mathrm{mg} / \mathrm{kg}$ i.v. as a muscle relaxant. The animal's body temperature was maintained at $37 \pm 0.5^{\circ} \mathrm{C}$ with an automatic heating device (model 73A-YSI; Yellow Springs Instruments, Yellow Springs, $\mathrm{OH}$ ). The femoral artery was cannulated for continuous monitoring of arterial blood pressure and arterial blood gas levels.

\section{Cerebral blood flow measurement}

A midline incision was made to expose the skull and cervical spine. A microsurgical drill was then used to create a $2.5-\mathrm{mm}$-diameter burr hole $6 \mathrm{~mm}$ lateral and $1 \mathrm{~mm}$ posterior to the bregma, as previously described [27]. To ensure reproducibility of recording, this location was held constant. Great care was taken to maintain dural integrity. Real-time cortical blood flow was then recorded continuously by using a laser Doppler flowmeter (LDF) (Laserflow BMP2; Vasamedics, Inc., Little Canada, MN). Using the stereotactic apparatus, a 2-mm-diameter probe was introduced into the burr hole. Great care was taken to maintain pial membrane integrity. The probe was not cemented into place, as there was no significant movement in this preparation. All data were collected and recorded on a Macintosh computer using standard charting software (Chart V4.1.1; ADInstruments; Mountain View, CA).

\section{Spinal cord stimulation}

The animal was placed in a stereotactic frame (model 900; Kopf Instruments, Tujunga, CA). A midline incision was made from the occiput to the cervical spine. The first and second lamina were identified and cleared of paraspinous muscles. A laminectomy between $\mathrm{C} 1$ and $\mathrm{C} 2$ was performed, sparing the dura. Meticulous hemostasis was maintained to keep the dorsal surface of spinal dura dry for the duration of the experiment. A standard stimulation setup was used, which consisted of a stimulator (model S48; Grass Instruments, Quincy, MA), a constant-current unit (model CCU1-A; Grass Instruments) and an oscilloscope (model 5111-A; Tektronix, Gaithersburg, MD). A ground electrode was placed in the left proximal hindquarters of the rat. A 1-mm platinum-ball electrode mounted on a stereotactic electrode holder was then lowered onto the exposed dorsal dural surface in the midline. After a stable LDF value baseline was established, monopolar electrical simulation was performed.

\section{SCS parameter testing protocol}

\section{Amplitude}

In the first series of experiments, we examined the effect of SCS amplitude on CBF response. Prior experiments have determined that the motor threshold in the rat is approximately $1.8 \mathrm{~mA}$ (unpublished data). Since it is unlikely that stimulation amplitudes exceeding motor threshold would be therapeutically meaningful, we selected $1.8 \mathrm{~mA}$ as the maximum amplitude tested. Setting the pulse width and frequency at the previously described settings of $0.1 \mathrm{~ms}$ and $50 \mathrm{~Hz}$ [30], we varied the stimulation amplitude. Each animal underwent three 2-minute stimulation periods, each followed by a rest period of $8-12$ minutes. Stimulation amplitudes were chose randomly amongst $0.1,0.4,0.8,1.2,1.5$ and $1.8 \mathrm{~mA}$. LDF was measured continuously, and recorded for the entire duration of each experiment.

\section{Pulse width}

Utilizing the stimulation amplitude found to elicit the most robust increase in LDF values, we tested the effect of various stimulation pulse width values between 0.1 to $0.5 \mathrm{~ms}$, while fixing the frequency at $50 \mathrm{~Hz}$. The range of tested pulse widths is well-within clinically utilized parameters in SCS. Once again, the stimulation period was 2 minutes and the rest interval between each of the three stimulation periods was 8-12 minutes. $\mathrm{CBF}$ was measured continuously in a fashion similar to that used in the first experimental series.

\section{Frequency}

The third series of experiments examined the effect of stimulation frequency on the CBF response. Utilizing the pulse width and amplitude found to elicit the most robust increase in LDF values, we tested the effect of various stimulation frequencies between 25 and $100 \mathrm{~Hz}$. As above, the stimulation duration was 2 minutes with an interval of 8-12 minutes between each of the three stimulation periods. LDF values were recorded for the entire duration of the experiment.

\section{Duration}

The issue of tachyphylaxis is a crucial one in determining the clinical utility of SCS in cerebral ischemia. Within the framework of this experimental stimulation model, it is not possible to test the effect of prolonged stimulation (e.g., hours, days). Therefore, we investigated the durability of the SCS response up to a maximal duration of 20 minutes. With those basic SCS parameters (pulse width, amplitude and frequency) obtained to elicit the most robust increase in LDF values, we extended the stimulation duration from 2 minutes to 5,10 or 20 minutes. LDF values were recorded for the entire duration of each experiment.

\section{Statistical analysis}

Changes in LDF measurements were expressed as percentages of baseline values $(\Delta \% \mathrm{LDF})$ and presented as mean \pm standard error of 
Table 1. Mean $\triangle \%$ LDFs in response to stimulation amplitudes

\begin{tabular}{lllcccc}
\hline Amplitude & $0.1 \mathrm{~mA}$ & $0.4 \mathrm{~mA}$ & $0.8 \mathrm{~mA}$ & $1.2 \mathrm{~mA}$ & $1.5 \mathrm{~mA}$ & $1.8 \mathrm{~mA}$ \\
\hline $\mathrm{n}$ & 8 & 7 & 6 & 10 & 6 & 7 \\
SCS & $0.3 \pm 0.4 \%$ & $2.6 \pm 0.7 \%^{*}$ & $11.8 \pm 1.3 \% *$ & $37.3 \pm 2.5 \% *$ & $49.0 \pm 2.5 \% *, \#$ & $45.2 \pm 3.7 \%^{*}$ \\
Post-SCS & $0.4 \pm 0.5 \%$ & $3.2 \pm 1.0 \%$ & $1.3 \pm 1.4 \%$ & $9.8 \pm 1.6 \%$ & $7.8 \pm 3.0 \%$ & $12.0 \pm 2.6 \%$ \\
\hline
\end{tabular}

* $P<0.01$ vs. baseline; $\# \quad P<0.001$ vs. $0.1,0.4,0.8$ and $1.2 \mathrm{~mA}$ groups.

$\triangle \%$ LDF: Cerebral blood flow changes compared to the baseline measured by the laser Doppler flowmetry; SCS: during the 2-minute stimulation period; Post-SCS: in 8 minutes after the stimulation. The stimulation frequency and pulse width are set at $50 \mathrm{~Hz}$ and $0.1 \mathrm{~ms}$, respectively. Values are expressed as mean $\pm \mathrm{SE}$.

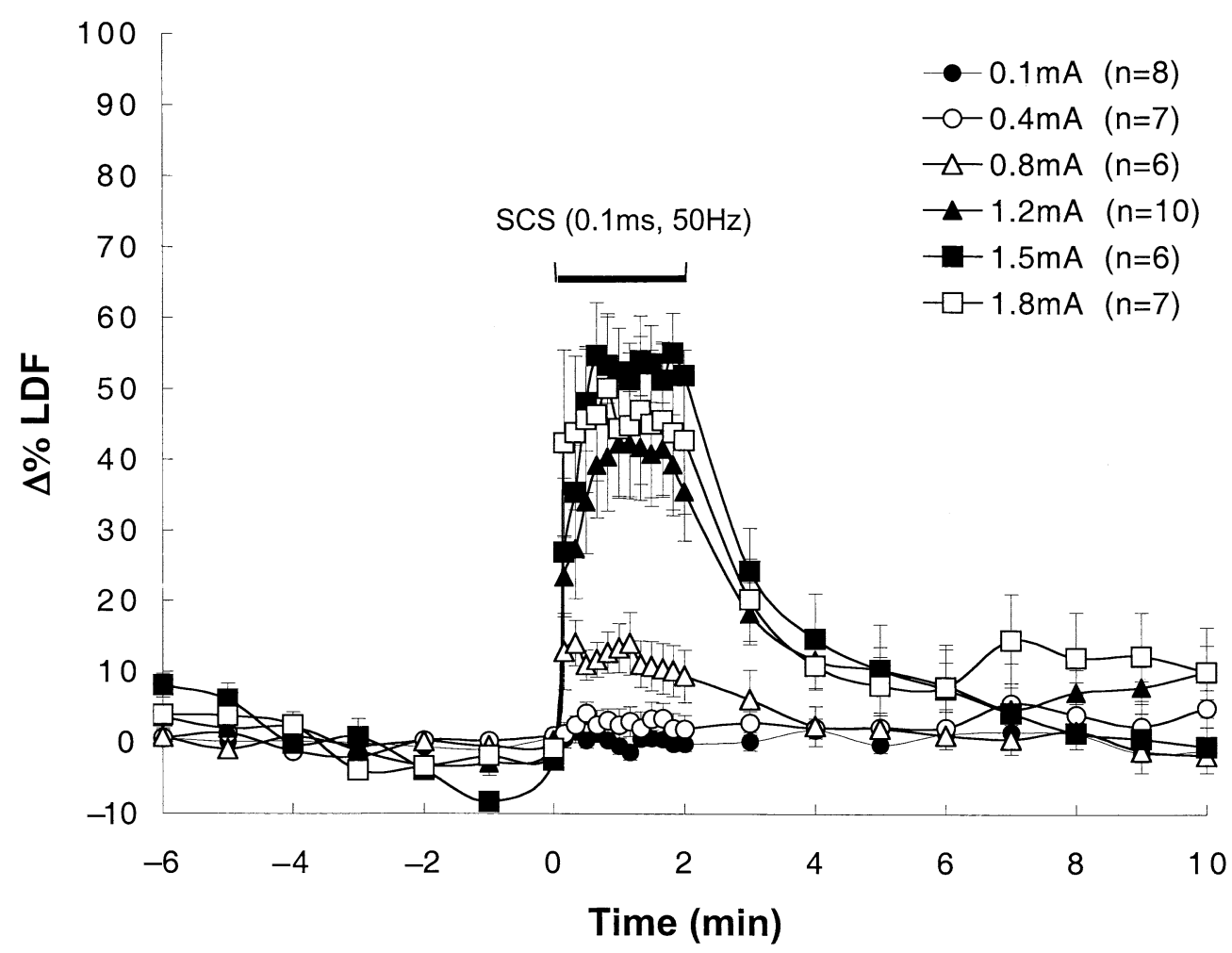

Fig. 1. Graph showing the effect of stimulation amplitude on LDF changes. The stimulation frequency and pulse width are set at $50 \mathrm{~Hz}$ and $0.1 \mathrm{~ms}$, respectively. $\Delta \%$ LDF: the percentage CBF change compared to the baseline measured by LDF. Values are expressed as mean $\pm \mathrm{SE}$

the mean. $\Delta \%$ LDFs in both of the stimulation and rest periods were averaged. Mean $\Delta \%$ LDFs in different groups were compared. Comparisons of values were made by using the two-tailed unpaired Student $t$-test and single factor ANOVA with post-hoc analysis using Bonferroni/Dunn test. Statistical significance was accepted at $P<0.05$.

\section{Results}

\section{Effect of SCS amplitude}

SCS induced a robust increase in cerebral LDF values, consistent with previous findings. The degree of LDF
Table 2. Mean $4 \%$ LDFs in response to stimulation pulse widths

\begin{tabular}{lccc}
\hline Pulse width & $0.1 \mathrm{~ms}$ & $0.25 \mathrm{~ms}$ & $0.5 \mathrm{~ms}$ \\
\hline $\mathrm{n}$ & 6 & 14 & 6 \\
$\mathrm{SCS}$ & $49.0 \pm 2.5 \%^{*}$ & $67.6 \pm 2.7 \%^{*}, \#$ & $22.0 \pm 2.5 \% *$ \\
Post-SCS & $7.8 \pm 3.0 \%$ & $5.9 \pm 1.9 \%$ & $9.5 \pm 2.6 \%$ \\
\hline
\end{tabular}

* $P<0.001$ vs. baseline; \# $P<0.001$ vs. other pulse width groups. $\Delta \%$ LDF: Cerebral blood flow changes compared to the baseline measured by the laser Doppler flowmetry; SCS: during the 2-minute stimulation period; Post-SCS: in 8 minutes after the stimulation. The frequency and amplitude are set at $50 \mathrm{~Hz}$ and $1.5 \mathrm{~mA}$, respectively. Values are expressed as mean $\pm \mathrm{SE}$. 


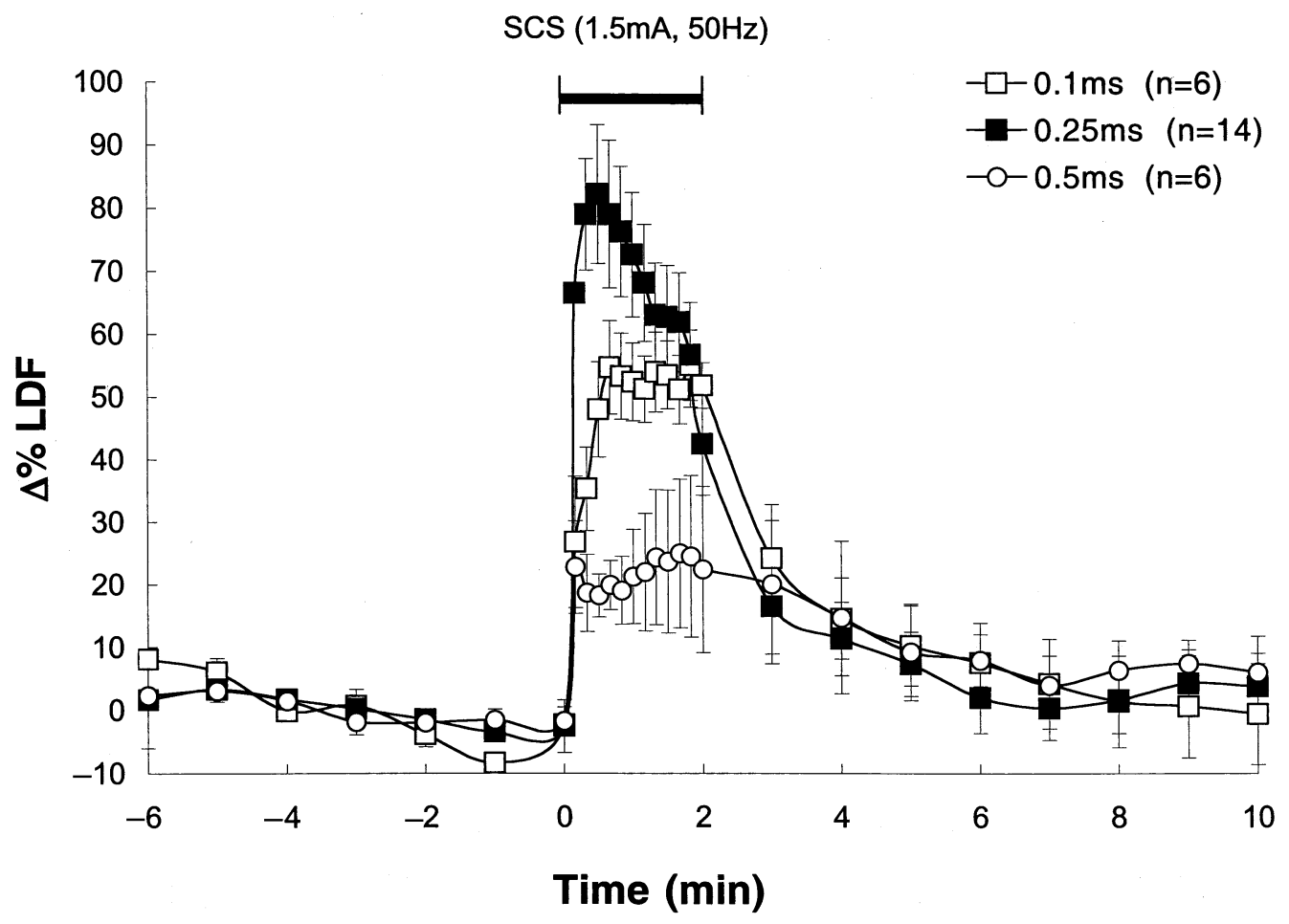

Fig. 2. Graph illustrating the effect of stimulation pulse width on LDF changes. The stimulation frequency and amplitude are set at $50 \mathrm{~Hz}$ and $1.5 \mathrm{~mA}$, respectively. $\triangle \%$ LDF: the percentage CBF change compared to the baseline measured by LDF. Values are expressed as mean $\pm \mathrm{SE}$

augmentation varied in a dose-dependent fashion with stimulation amplitude. At the lowest SCS amplitude tested $(0.1 \mathrm{~mA})$, there was no significant increase in LDF values. In animals undergoing stimulation at the current amplitude of $1.5 \mathrm{~mA}$, the average increase in LDF value was approximately $50 \%$ over baseline $(P<0.001)$. This stimulation amplitude induced the largest increase in LDF value in the range of amplitudes tested (Table 1 and Fig. 1).

\section{Effect of SCS pulse width}

In this series of experiments, we held stimulation amplitude and frequency constant at $1.5 \mathrm{~mA}$ and $50 \mathrm{~Hz}$, respectively. When varying the pulse width between 0.1 and $0.5 \mathrm{~ms}$, we noted that the maximal increase in LDF values occurred in the 0.25 -ms group. In those animals undergoing SCS with a 0.25 -ms pulse width, the average increase in LDF was $67.6 \pm 3.3 \%(P<0.001$ vs. other groups) over the 2-minute stimulation period, with a mean peak value of greater than $80 \%$. Increasing pulse width to $0.5 \mathrm{~ms}$ did not improve the $\mathrm{CBF}$ response (Table 2 and Fig. 2).

\section{Effect of SCS frequency}

We examined the frequency modulation SCS effect on the cerebrovascular response by holding the stimulation amplitude and pulse width constant at $1.5 \mathrm{~mA}$ and $0.25 \mathrm{~ms}$, respectively. When the stimulation frequency varied from 25 to $100 \mathrm{~Hz}$, the maximal increase in LDF value still occurred in the $50-\mathrm{Hz}$ group $(P<0.001$ vs. other groups). Mean $\Delta \%$ LDFs in the 2-minute SCS and following 8-minute rest period are shown in Table 3. The time course of mean $\Delta \%$ LDF using different

Table 3. Mean $4 \%$ LDFs in response to stimulation frequencies

\begin{tabular}{lccc}
\hline Frequency & $25 \mathrm{~Hz}$ & $50 \mathrm{~Hz}$ & $100 \mathrm{~Hz}$ \\
\hline $\mathrm{n}$ & 6 & 14 & 8 \\
$\mathrm{SCS}$ & $21.2 \pm 2.0 \%^{*}$ & $67.6 \pm 2.7 \% *, \#$ & $26.6 \pm 1.7 \% *$ \\
Post-SCS & $2.3 \pm 5.9 \%$ & $5.9 \pm 1.9 \%$ & $0.5 \pm 1.8 \%$ \\
\hline
\end{tabular}

* $P<0.001$ vs. baseline; \# $P<0.001$ vs. other frequency groups. $\Delta \%$ LDF: Cerebral blood flow changes compared to the baseline measured by the laser Doppler flowmetry; SCS: during the 2-min stimulation period; Post-SCS: in $8 \mathrm{~min}$ after the stimulation. The stimulation amplitude and pulse width are set at $1.5 \mathrm{~mA}$ and $0.25 \mathrm{~ms}$, respectively. Values are expressed as mean $\pm \mathrm{SE}$. 


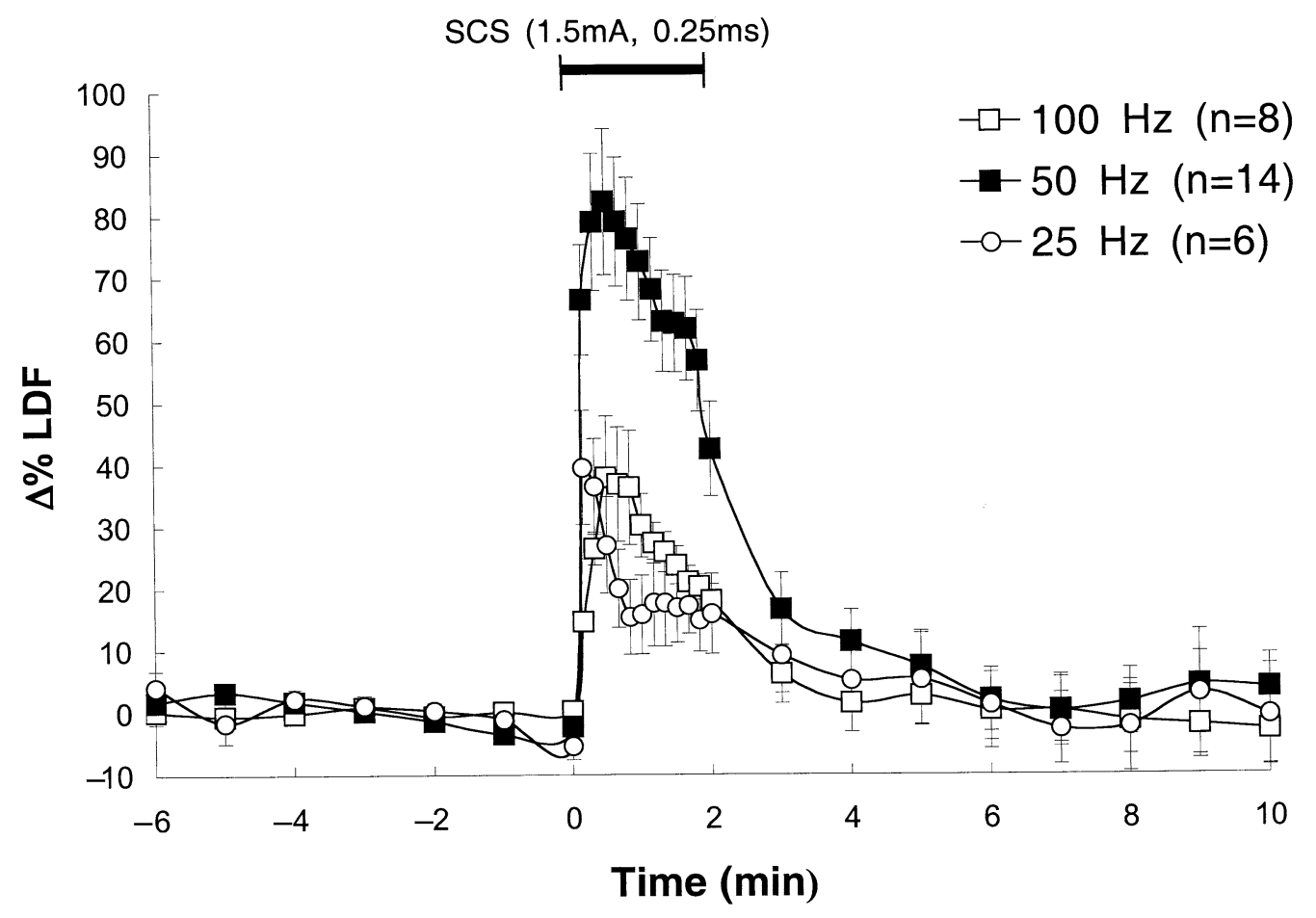

Fig. 3. The effect of stimulation frequency on LDF changes. The stimulation pulse width and amplitude are set at $0.25 \mathrm{~ms}$ and $1.5 \mathrm{~mA}$, respectively. $\Delta \%$ LDF: the percentage CBF change compared to the baseline measured by LDF. Values are expressed as mean $\pm \mathrm{SE}$

Table 4. Mean $\triangle \%$ LDFs in response to stimulation durations

\begin{tabular}{|c|c|c|c|c|}
\hline SCS-Duration & 2 minutes & 5 minutes & 10 minutes & 20 minutes \\
\hline $\mathrm{n}$ & 14 & 6 & 14 & 6 \\
\hline SCS & $67.6 \pm 2.7 \% *$ & $66.2 \pm 3.4 \% *$ & $61.7 \pm 2.4 \% *$ & $52.4 \pm 2.3 \%{ }^{*}, \#$ \\
\hline Post-SCS & $5.9 \pm 1.9 \%{ }^{\S}$ & $9.7 \pm 3.1 \%^{\S}$ & $9.1 \pm 1.6 \%{ }^{\S}$ & $-2.7 \pm 1.6 \%$ \\
\hline
\end{tabular}

* $P<0.001$ vs. baseline; ${ }^{\S} P<0.001$ vs. baseline; ${ }^{\#} P<0.001$ vs. other duration groups.

$\triangle \%$ LDF: Cerebral blood flow changes compared to the baseline measured by the laser Doppler flowmetry; SCS: during the stimulation period; PostSCS: in 8 minutes after the stimulation. The stimulation amplitude, frequency and pulse width are set at $1.5 \mathrm{~mA}, 50 \mathrm{~Hz}$ and $0.25 \mathrm{~ms}$, respectively. Values are expressed as mean $\pm \mathrm{SE}$.

stimulation frequency during the entire experimental period is illustrated in Fig. 3.

\section{Effect of SCS duration}

Using stimulation parameters of 0.25 -ms pulse width, $1.5-\mathrm{mA}$ amplitude and $50-\mathrm{Hz}$ frequency and extending the SCS period up to 20 minutes, we observed a persistent and robust increase in cerebral LDF values during the entire stimulation period. The average value of $\Delta \% \mathrm{LDF}$ in consecutive 5-minute epochs was $60.0 \pm 1.6 \%, \quad 61.1 \pm 2.3 \%, \quad 53.2 \pm 5.2 \%$ and
$46.3 \pm 5.8 \%$, respectively. There was no statistically difference among the first three 5-minute epochs, but the response appeared to diminish somewhat in the last 5-minute epochs $(P<0.001$ vs. 1 st and 2 nd epoch). Mean $\Delta \%$ LDF values during and after the stimulation periods in various SCS-length groups are listed in Table 4. After stimulation, LDF values gradually returned toward baseline values, reaching a level within $10 \%$ of the original baseline by 8 minutes following the cessation of stimulation. Figure 4 delineates the time course of mean $\Delta \%$ LDFs in various SCS-length groups. 


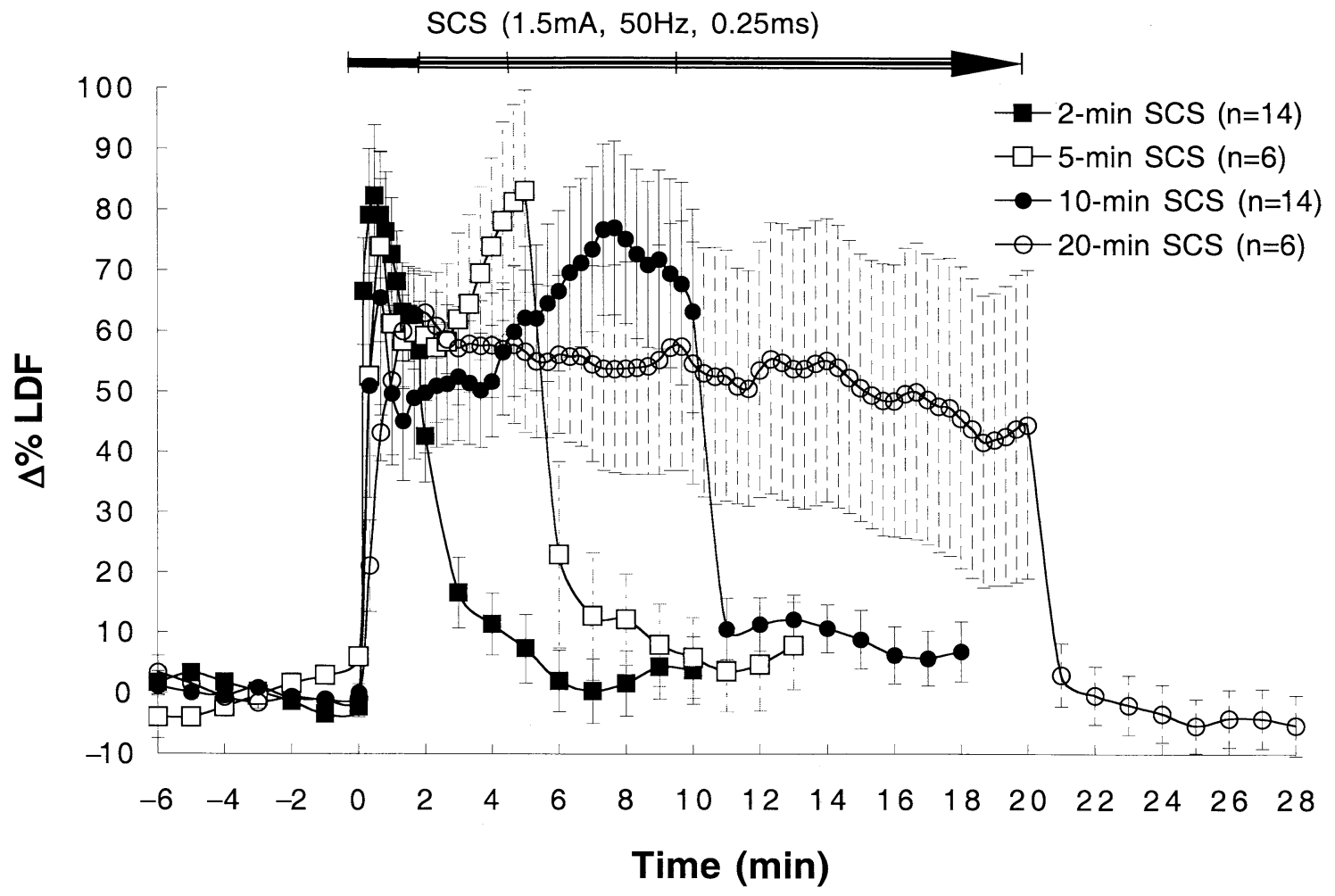

Fig. 4. The effect of stimulation duration on LDF changes. The stimulation pulse width, frequency and amplitude are set at $0.25 \mathrm{~ms}, 50 \mathrm{~Hz}$ and $1.5 \mathrm{~mA}$, respectively. $\triangle \%$ LDF: the percentage CBF change compared to the baseline measured by LDF. Values are expressed as mean $\pm \mathrm{SE}$

\section{Discussion}

Spinal cord stimulation has been used for the treatment of chronic pain over the last three decades. Clinical observations and recent experimental studies have suggested clinical utility of this modality in the treatment of ischemia as well as other conditions, such as early cerebral vasospasm [35], stroke [38], postapoplectic spastic hemiplegia [26] and prolonged coma [9]. The successful use of SCS in the treatment or prevention of ischemia hinges on an understanding of the underlying mechanisms, clinically relevant parameters and limitations of this technique.

The augmentative effect of SCS on CBF has been demonstrated in a number of animal models and early clinical studies. Previous studies have indicated that spinal cord stimulation results in an increase in cerebral blood flow in a number of animal models, and that this increase is dependent on the spinal level of stimulation. Previous studies in our laboratory have indicated that the SCS effect on CBF may involve indirect effects on centers in the brainstem as well as an alteration in sympathetic tone $[28,30]$. Sympathetic tone appears to play a major role in SCS-induced CBF augmentation [37], and this sympathetic response is mediated primarily by $\alpha_{1}$-adrenergic receptors [27]. These lines of evidence suggest that cervical SCS modulates activity within autonomic vasomotor nuclei in the brainstem, thereby increasing CBF [29].

As the use of SCS to increase blood flow becomes more widespread, it is necessary to delineate the effects of various stimulation parameters on this response and examine its durability. Up until now, few studies have examined the effects of altering stimulation parameters in the clinical setting [3]. Tulgar et al. [33] investigated 1,100 electrode combinations at various spinal levels. When utilizing $210 \mu$ s long rectangular monophasic pulses delivered at a constant frequency of $50 \mathrm{~Hz}$, the authors found that at the level of $\mathrm{C} 2-\mathrm{C} 3$, the perception and tolerance threshold were $1.23 \pm 0.09$ and $1.68 \pm 0.11 \mathrm{~mA}$, respectively. Miles [25] recommended keeping the treatment level half way between the perception threshold and the tolerance threshold. While no clinical study has examined SCS parameters in the CBF response, a limited experimental study has suggested that lower stimulation frequencies $(<200 \mathrm{~Hz})$ are associated with augmentation of CBF. In this study, Isono et al. [16] found that the stimulation frequencies of 200 and $2,000 \mathrm{~Hz}$ did not result in a CBF increase, whereas a moderately low frequency $(20 \mathrm{~Hz})$ increased CBF significantly. 
The present study extends and further defines the parameters of stimulation necessary to elicit a significant cerebrovascular response. The stimulation amplitude, pulse width and rate found to be associated with a maximal rise in cerebral LDF values are all well-within commonly utilized parameter ranges [36]. This study therefore supports the clinical utility of SCS in the augmentation of cerebral blood flow. Moreover, this study suggests that tachyphylaxis to the effect of SCS on CBF does not seem to limit its effectiveness in the experimental model. This finding is corroborated by previously described neuroprotection in a focal cerebral ischemia model [31].

In the present study, we employed LDF as a measurement to achieve a real time CBF recording. Although the use of LDF does not allow one to examine the spatial characteristics or absolute magnitude of the observed blood flow changes, this technique provides a continuous record of CBF changes during the SCS period as well as the pre- and post-SCS period. LDF has previously been validated against quantitative $\mathrm{CBF}$ measures in this model [31]. Investigation of the long-term effects of SCS on CBF requires its study in awake animals.

SCS provides an encouraging therapeutic option for the treatment and prevention of cerebral ischemia. The study of SCS in an experimental setting has allowed delineation of the likely underlying mechanism(s) and now provides some initial guideline in its clinical implementation. Further study of tachyphylaxis of the effect in awake animals will be required in order to fully realize the potential of this therapy before clinical studies can be pursued.

\section{Acknowledgement}

The authors would like to thank Dr. Richard Keep for his editorial assistance.

\section{Disclosure statement}

This work was supported by a grant from the National Institutes of Health to Dr. Sagher (RO1 NS41061).

\section{References}

1. Anderson DJ, Jenkins C, McInally C (1999) Using spinal cord stimulation to manage angina pain. DCCN - Dimensions of Critical Care Nursing 18(3): 12-13

2. Augustinsson LE, Eliasson T, Mannheimer C (1995) Spinal cord stimulation in severe angina pectoris. Stereotact Funct Neurosurg 65(1-4): 136-141
3. Barolat G, Zeme S, Ketcik B (1991) Multifactorial analysis of epidural spinal cord stimulation. Stereotact Funct Neurosurg 56(2): 77-103

4. Broseta J, García-March G, Sánchez-Ledesma MJ, Gonçalves J, Silva I, Barcia JA, Llácer JL, Barcia-Salorio JL (1994) Highcervical spinal cord electrical stimulation in brain low perfusion syndromes: Experimental basis and preliminary clinical report. Stereotact Funct Neurosurg 62(1-4): 171-178

5. Canavero S, Bonicalzi V (2003) Spinal cord stimulation for central pain. Pain 103(1-2): 225-226

6. Clavo B, Robaina F, Catala L, Valcarcel B, Morera J, Carames MA, Ruiz-Egea E, Panero F, Lloret M, Hernandez MA (2003) Increased locoregional blood flow in brain tumors after cervical spinal cord stimulation. J Neurosurg 98(6): 1263-1270

7. Cook AW, Oygar A, Baggenstos P, Pacheco S, Kleriga E (1976) Vascular disease of extremities. Electric stimulation of spinal cord and posterior roots. N Y State J Med 76(3): 366-368

8. de Jongste MJ, Hautvast RW, Hillege HL, Lie KI (1994) Efficacy of spinal cord stimulation as adjuvant therapy for intractable angina pectoris: a prospective, randomized clinical study. Working Group on Neurocardiology. J Am Coll Cardiol 23(7): 1592-1597

9. Fujii M, Sadamitsu D, Maekawa T, Uesugi S, Ozaki S, Koizumi H, Uetsuka S, Sakamoto K, Yamashita T, Ito H (1998) Spinal cord stimulation therapy at an early stage for unresponsive patients with hypoxic encephalopathy. No Shinkei Geka - Neurol Surg 26(4): 315-321

10. Garcia-March G, Sanchez-Ledesma MJ, Anaya J, Broseta J (1989) Cerebral and carotid haemodynamic changes following cervical spinal cord stimulation. An experimental study. Acta Neurochirurgica - Supplementum 46: 102-104

11. Ghajar AW, Miles JB (1998) The differential effect of the level of spinal cord stimulation on patients with advanced peripheral vascular disease in the lower limbs. Brit J Neurosurg 12(5): 402-408

12. Hautvast RW, Blanksma PK, DeJongste MJ, Pruim J, van der Wall EE, Vaalburg W, Lie KI (1996) Effect of spinal cord stimulation on myocardial blood flow assessed by positron emission tomography in patients with refractory angina pectoris. Am J Cardiol 77(7): 462-467

13. Hautvast RW, DeJongste MJ, Staal MJ, van Gilst WH, Lie KI (1998) Spinal cord stimulation in chronic intractable angina pectoris: a randomized, controlled efficacy study. American Heart Journal 136(6): 1114-1120

14. Horsch S, Claeys L (1994) Epidural spinal cord stimulation in the treatment of severe peripheral arterial occlusive disease. Ann Vasc Surg 8(5): 468-474

15. Hosobuchi Y (1985) Electrical stimulation of the cervical spinal cord increases cerebral blood flow in humans. Appl Neurophysiol 48(1-6): 372-376

16. Isono M, Kaga A, Fujiki M, Mori T, Hori S (1995) Effect of spinal cord stimulation on cerebral blood flow in cats. Stereotact Funct Neurosurg 64(1): 40-46

17. Kay AD, McIntyre MD, Macrae WA, Varma TR (2001) Spinal cord stimulation - a long-term evaluation in patients with chronic pain. Brit J Neurosurg 15(4): 335-341

18. Kumar K, Toth C, Nath RK, Verma AK, Burgess JJ (1997) Improvement of limb circulation in peripheral vascular disease using epidural spinal cord stimulation: a prospective study. J Neurosurg 86(4): 662-669

19. Linderoth B, Fedorcsak I, Meyerson BA (1991) Peripheral vasodilatation after spinal cord stimulation: animal studies of putative effector mechanisms. Neurosurg 28(2): 187-195

20. Linderoth B, Herregodts P, Meyerson BA (1994) Sympathetic mediation of peripheral vasodilation induced by spinal cord 
stimulation: animal studies of the role of cholinergic and adrenergic receptor subtypes. Neurosurg 35(4): 711-719

21. Matsui T, Hosobuchi Y (1989) The effects of cervical spinal cord stimulation (cSCS) on experimental stroke. Pacing Clin Electrophysiol 12(4 Pt 2): 726-732

22. Meglio M, Cioni B, Visocchi M (1991) Cerebral hemodynamics during spinal cord stimulation. Pacing Clin Electrophysiol 14(1): $127-130$

23. Meglio M, Cioni B, Visocchi M, Nobili F, Rodriguez G, Rosadini G, Chiappini F, Sandric S (1991) Spinal cord stimulation and cerebral haemodynamics. Acta Neurochirurgica 111(1-2): $43-48$

24. Melzack R, Wall PD (1965) Pain mechanisms: a new theory. Science 150(699): 971-979

25. Miles J (1984) Electrical stimulation for the relief of pain. Ann R Coll Surg Engl 66(2): 108-112

26. Nakamura S, Tsubokawa T (1985) Evaluation of spinal cord stimulation for postapoplectic spastic hemiplegia. Neurosurg 17(2): 253-259

27. Patel S, Huang DL, Sagher O (2003) Sympathetic mechanisms in cerebral blood flow alterations induced by spinal cord stimulation. J Neurosurg 99(4): 754-761

28. Patel S, Huang DL, Sagher O (2004) Evidence for a central pathway in the cerebrovascular effects of SCS. Neurosurg (in press)

29. Reis DJ, Golanov EV (1997) Autonomic and vasomotor regulation. International Review of Neurobiology 41: 121-149

30. Sagher O, Huang DL (2000) Effects of cervical spinal cord stimulation on cerebral blood flow in the rat. J Neurosurg 93(1 Suppl): $71-76$

31. Sagher O, Huang DL, Keep RF (2003) Spinal cord stimulation reducing infarct volume in a model of focal cerebral ischemia in rats. J Neurosurg 99(1): 131-137

32. Tallis RC, Illis LS, Sedgwick EM, Hardwidge C, Garfield JS (1983) Spinal cord stimulation in peripheral vascular disease. J Neurol Neurosurg Psychiatry 46(6): 478-484

33. Tulgar M, Barolat G, Ketcik B (1993) Analysis of parameters for epidural spinal cord stimulation. 1. Perception and tolerance thresholds resulting from 1,100 combinations. Stereotact Funct Neurosurg 61(3): 129-139

34. Ubbink DT, Spincemaille GH, Prins MH, Reneman RS, Jacobs MJ (1999) Microcirculatory investigations to determine the effect of spinal cord stimulation for critical leg ischemia: the Dutch multicenter randomized controlled trial. J Vasc Surg 30(2): 236-244

35. Visocchi M, Argiolas L, Meglio M, Cioni B, Basso PD, Rollo M, Cabezas D (2001) Spinal cord stimulation and early experimental cerebral spasm: the "functional monitoring" and the "preventing effect". Acta Neurochirurgica 143(2): 177-185

36. Visocchi M, Cioni B, Pentimalli L, Meglio M (1994) Increase of cerebral blood flow and improvement of brain motor control following spinal cord stimulation in ischemic spastic hemiparesis. Stereotact Funct Neurosurg 62(1-4): 103-107

37. Visocchi M, Cioni B, Vergari S, Marano G, Pentimalli L, Meglio M (1994) Spinal cord stimulation and cerebral blood flow: An experimental study. Stereotact Funct Neurosurg 62(1-4): 186-190

38. Visocchi M, Giordano A, Calcagni M, Cioni B, Di Rocco F, Meglio M (2001) Spinal cord stimulation and cerebral blood flow in stroke: personal experience. Stereotact Funct Neurosurg 76(3-4): 262-268

39. Visocchi M, Tartaglione T, Romani R, Meglio M (2001) Spinal cord stimulation prevents the effects of combined experimental ischemic and traumatic brain injury. An MR study. Stereotact Funct Neurosurg 76(3-4): 276-281

\section{Comments}

This is an experimental study on the effect of cervical spinal cord stimulation (SCS) on cerebral blood flow augmentation. The authors have investigated various stimulation parameters (amplitude, pulse width and frequency) to find out the optimal setting for the most pronounced effect on CBF.

In addition, at least in this animal model, an increase in CBF was noted as long as the stimulation was continued with no signs of tachyphylaxis.

This is a well designed and carefully conducted study providing some useful information on the increase of CBF elicited by SCS.

The observation that SCS results in increased peripheral and central blood flow has been around for some time. In fact, it has been used clinically for the treatment of angina pectoris and peripheral ischaemic disease. So far, it has not been used on a large scale for cerebral ischaemic conditions. The reason for this may be that we still do not know the mechanism of action how SCS leads to an increase of CBF.

In addition, it is a rather invasive method thereby preventing it's widespread clinical application. However, one could imagine that the application of this technique might be tried in patients with vasospasm following $\mathrm{SAH}$.

Peter Schmiedek Mannheim

Spinal cord stimulation (SCS) affects peripheral, coronaric and cerebral blood flow (CBF) in humans. Functional reversible sympathectomy may be one of the mechanisms put in play by SCS to produce such effects. One of the most fascinating effects of SCS is the possibility to interfere with the mechanisms of regulation of the cardiovascular system.

In this paper the Authors investigate on the parameters influencing augmentation of CBF by SCS. The aim is to increase the interest in SCS as possible therapy (treatment and prevention) of cerebral ischaemia in humans. The paper has some limits related to the choice of an animal (rat) target rather than the human', the type of CBF assessment (not global but unilateral CBF investigation) but deserves consideration because confirm the interest on neurosurgeons on this topic.

Massimiliano Visocchi

Rome

Correspondence: Oren Sagher, M.D., Department of Neurosurgery, University of Michigan Health System, 1500 E. Medical Center Drive, Ann Arbor, MI 48109-0338. e-mail: osagher@umich.edu 